\title{
Hominis Placenta facilitates hair re-growth by upregulating cellular proliferation and expression of fibroblast growth factor-7
}

Hyung-Sik Seo ${ }^{1}$, Dong-Jin Lee ${ }^{2}$, Jae-ho Chung ${ }^{3}$, Chang-Hyun Lee ${ }^{4}$, Ha Rim Kim5, Jae Eun Kim${ }^{6}$, Byung Joo Kim, Myeong Ho Jung ${ }^{7,8}$, Ki-Tae Ha ${ }^{9}$ and Han-Sol Jeong ${ }^{9^{*}}$

\begin{abstract}
Background: Hominis Placenta (HP) known as a restorative medicine in Traditional Chinese Medicine (TCM), has been widely applied in the clinics of Korea and China as an anti-aging agent to enhance the regeneration of tissue. This study was conducted to investigate whether topical treatment of HP promotes hair regrowth in the animal model.

Methods: The dorsal hairs of 8-week-old C57BL/6 mice were depilated to synchronize hair follicles to the anagen phase. HP was applied topically once a day for 15 days. Hair growth was evaluated visually and microscopically. The incorporation of bromodeoxyuridine (BrdU) and expression of proliferating cell nuclear antigen (PCNA), fibroblast growth factor-7 (FGF-7) in dorsal skin tissue was examined by immunohistochemical analysis. Reverse transcription polymerase chain reaction (RT-PCR) was used to measure the mRNA expression of FGF-7.

Results: HP exhibited potent hair growth-promoting activity in C57BL/6 mice. Gross examination indicated that HP markedly increased hair regrowth as well as hair density and diameter. Histologic analysis showed that HP treatment enhanced the anagen induction of hair follicles.

Immunohistochemical analysis revealed that BrdU incorporation and the expressions of PCNA were increased by treatment of HP. HP treatment significantly increased the expression of FGF-7, which plays pivotal roles to maintain anagen phase both protein and mRNA levels.
\end{abstract}

Conclusions: Taken together, our results indicate that HP has a potent hair growth-promoting activity; therefore, it may be a good candidate for the treatment of alopecia.

Keywords: Hominis Placenta, Hair growth, Anagen, Bromodeoxyuridine, Fibroblast growth factor-7

\section{Background}

The number of patients with alopecia is constantly increasing because of the stressful modern lifestyle. In a vast majority of cases, hair loss is attributable to hormones and genetic factors [1, 2]; however, other factors, such as autoimmune diseases, medications, and physiologic and psychological stresses have been linked to hair loss [3-5]. These causative factors can alter the hair follicle cycle.

\footnotetext{
* Correspondence: jhsol33@pusan.ac.kr

${ }^{9}$ Division of Applied Medicine, School of Korean Medicine, Pusan National University, Yangsan, Republic of Korea

Full list of author information is available at the end of the article
}

Humans have about 5 million hair follicles at birth and new follicles do not develop after birth [6]. Each hair follicle, which produce hair shafts, undergo successive cycles consisting of anagen (growing phase), catagen (transitional phase), and telogen (resting phase). This cycling occurs over the lifetime of the individual. Initiation and progression of the hair follicle cycle are determined by intercellular communications between the dermis and surface epithelium [7]. However, the complex molecular interactions between the cells of the hair follicle are not fully understood and the exact cause of alopecia has not been elucidated [8].

Androgenic alopecia (AA) which is the most common form of hair loss in men is characterized by thinning 
and visible loss of frontal hair. Under the influence of dihydrotestosterone (DHT), terminal hair follicles gradually change to miniaturized follicles. Hair follicular miniaturization arises as a consequence of progressive shortening of the duration of successive anagen [9]. Therefore, extension of anagen phase could be a good strategy for the treatment of hair loss such as AA.

Hominis placenta (HP) has been used in Korea and China to improve general well-being. Since the 1950s, HP has been used as therapeutic agent. By stimulation of liver regeneration, and controlling of endocrine system, HP has been applied to the liver diseases [10], and climacteric symptoms [11]. In addition, placenta extract was known to have antioxidant, anti-inflammatory, wound healing, and nerve growth-promoting effects [12-15]. Therefore, it is possible that the various effects of HP could influence the hair growth cycle.

One clinical study has already drawn attention to the hair-growth promoting effects of placental extract [16]. More recently, there was another report about hair growth promoting effects of cow placenta [17]. In this study, we investigated the hair growth-promoting effect of HP by measuring the cellular proliferation and expression of FGF-7.

\section{Methods}

\section{Materials}

Human placental extracts was provided from the Korean Pharmacopuncture Institute (Seoul, Korea). Primary antibodies specific for bromodeoxyuridine (BrdU), proliferating cell nuclear antigen (PCNA), fibroblast growth factor-7 (FGF-7), ß-actin were purchased from Santa Cruz Biotechnology, Inc. (Santa Cruz, CA, USA). Biotinylated goat anti-mouse immunoglobulin G (IgG) and avidin-biotin peroxidase complex were purchased from Vector Laboratories, Inc. (Burlingame, CA, USA). HRPlinked anti-mouse Ig $G$ antibody was purchased from GE Healthcare Life Science (Pittsburgh, PA, USA). 3-3'diaminobenzidine, PBS solution, and hydrogen peroxide were purchased from Sigma-Aldrich Co. (Youngin, Korea). Trizol reagent and a reverse transcriptase-polymerase chain reaction (RT-PCR) kit were purchased from Invitrogen (Carlslab, CA, USA) and Bioneer Co. (Daejeon, Korea), respectively.

\section{Animals and in vivo hair growth activity}

Seven-week-old male C57BL/6 mice were purchased from Samtaco Bio Korea, Ltd. (Osan, Korea) and allowed to adapt to the new environment for one week. The mice were housed in certified, standard laboratory cages and were provided with food and water ad libitum prior to the experiment. Amount of foods that consumed by one mouse per one week was about $42 \mathrm{~g}$, and the amount of drinking water in the same condition was about $60 \mathrm{ml}$. Fifteen mice were divided into the following three groups (5 mice per group): normal saline-, $5 \%$ minoxidil-, and HP-treated groups. The animal protocol used in this study was reviewed by the Pusan National University-Institutional Animal Care and Use Committee (PNU-IACUC) according to their ethical and scientific procedures and was approved (Approval Number PNU-2014-0581).

This study was designed according to the guidelines of the Korean Food and Drug Administration (KFDA) to evaluate hair growth promoting efficacy. The dorsal hairs of 8-week-old C57BL/6 mice, with hair follicles in the telogen phase of the hair growth cycle, were depilated to synchronize hair follicle growth to the anagen phase. One day after depilation, the mice were topically treated with normal saline, $5 \%$ minoxidil, or HP once a day for 15 days (Fig. 1). Hair growth and thickness in the depilated dorsal skin lesions were measured by dermoscopy (Sometech, Inc., Seoul, Korea).

\section{Skin histology}

C57BL/6 mice were euthanized at $16^{\text {th }}$ day after depilation. Dorsal depilated skin tissues were collected from the mice and fixed for $24 \mathrm{~h}$ at room temperature in

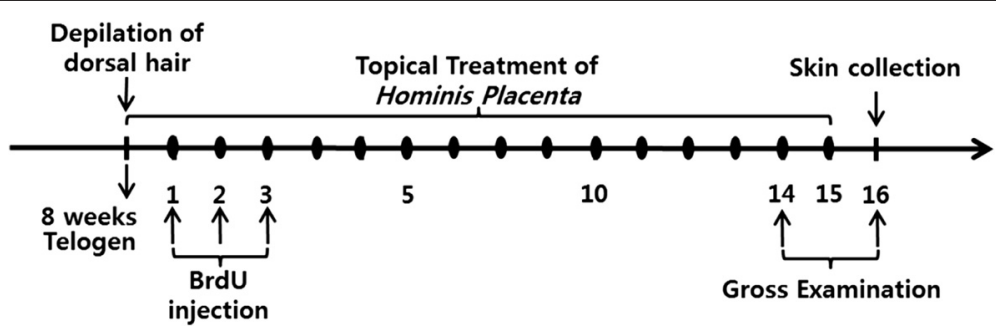

Fig. 1 Experimental scheme. Dorsal hairs of 8-week-old C57BL/6 mice, which were in the telogen phase of the hair cycle, were depilated to synchronize anagen induction. The mice were divided into following three groups: group 1, normal saline-treated negative control; group 2, $5 \%$ minoxidil-treated positive control; group 3, Hominis Placenta (HP)-treated experimental group ( $n=5$ per group). Each agent were topically applied from the 1st to the 15th day after hair depilation. Bromodeoxyuridine (BrdU) was administered twice a day on the 1st, 2nd, and the 3rd day after depilation. Depilated dorsal skin lesions were collected at the 16th day after depilation and stained with hematoxylin and eosin. $\bullet$, Topical treatment with normal saline, $5 \%$ minoxidil, or HP 
Bouin's solution. After dehydration, the skin tissues were embedded in paraffin, cut into 7 - $\mu$ m-thick sections, and placed onto glass slides. The slides were de-paraffinized with xylene and stained with hematoxylin and eosin (H\&E). Processed skin tissues were examined by light microscopy (Carl Zeiss, Germany).

\section{Immunohistochemistry}

BrdU, which is commonly used to label proliferating cells in tissue sections [18], was injected intraperitoneally at $50 \mu \mathrm{g} / \mathrm{g}$ b.w. twice daily for three consecutive days after hair depilation as described in a previous study [19]. Dorsal skin tissues were collected 16 days after the first treatment and subjected to immunostaining. Permeabilized tissue sections were incubated with the following antibodies by using the dilutions indicated in the manufacturer's instructions: mouse anti-BrdU (1:200), anti-PCNA (1:1000), and anti-FGF-7 (1:200). After the primary antibody incubation, skin tissues were incubated with biotinylated goat anti-mouse IgG in a moisture chamber for $30 \mathrm{~min}$. After washing, the tissues were incubated with HRP-conjugated avidin-biotin complex. After two more washing steps, the tissues were incubated with $30 \mathrm{mg}$ of 3-3' diaminobenzidine dissolved in $150 \mathrm{~mL}$ of $0.1 \mathrm{M}$ PBS solution for another $5 \mathrm{~min}$, and then $0.005 \%$ hydrogen peroxide was added for $15 \mathrm{~min}$ to develop the color. Immunoreactivity was examined by light microscopy.

\section{RT-PCR}

Total RNA was isolated using TRIzol reagent, and complementary DNA (cDNA) was synthesized using AccuPower RT PreMix (Bioneer, Daejon, Korea) according to the manufacturer's instructions. Specific DNA sequences were amplified with AccuPower PCR PreMix (Bioneer, Daejon, Korea). The oligonucleotide primer sequences were as follows: FGF-7, forward 5'-AGATCATGCTTCCACCT CGT-3' and reverse 5' -TGGGTCCCTTTCACTTTGCC3'; GAPDH, forward 5'-GGAGCCAAAAGGGTCATCA 'T-3' and reverse 5'-GTGATGGCATGGACTGTGGT-3'. Amplified products were separated on $1.0 \%$ agarose gels and analyzed under ultraviolet light. Images were captured using a GelDoc-It TS Imaging System (UVP, LLC, Upland, CA, USA).

\section{Western blot}

To isolate proteins, skin tissues were lysed by RIPA buffer $(0.1 \%$ SDS, 1 \% NP-40, 50 mM Tris, pH7.5, $150 \mathrm{mM}$ $\mathrm{NaCl}, 50 \mathrm{mM} \mathrm{NaF}, 1 \mathrm{mM}$ EDTA). Protein concentration of cell lysate was determined by Bradford method. After denaturation of protein at $100{ }^{\circ} \mathrm{C}$ for $5 \mathrm{~min}, 10 \mu \mathrm{g}$ of proteins were loaded on the $10 \%$ polyacrylamide gel, then separated by electrophoresis. After separation, the proteins were transferred to the polyvinylidene difluoride
(PVDF) membrane. The membrane was blocked by $5 \%$ non-fat milk in TBST for $1 \mathrm{~h}$ at room temperature. After blocking, the membrane was washed by TBST 3 times, then incubated with 1: 500 diluted mouse monoclonal anti-FGF7 antibody at $4{ }^{\circ} \mathrm{C}$ for overnight. The next day, the membrane was washed 3 times by TBST, then incubated with anti-mouse Ig G HRP-linked antibody for $1 \mathrm{~h}$, at room temperature. After finishing another 3 times of washing, enhanced chemilumescent (ECL) substrates were added to the membrane for the detection of antibody. Images were captured by using Amersham Imager 600 (Pittsburgh, PA, USA).

\section{Statistical analyses}

Data are expressed as the mean \pm standard deviation (SD). Statistical differences between means were determined by one-way analysis of variance (ANOVA) for repeated measures. $P$ values less than 0.05 were considered significant.

\section{Results \\ HP promotes hair re-growth on the depilated skin lesions of C57BL/6 mice}

We tested the hair growth-promoting activity of HP. HP was applied topically daily onto the depilated dorsal lesions of C57BL/6 mice. Normal saline, $5 \%$ minoxidil were used as the negative and positive control, respectively (Fig. 2, left and middle panel). HP promoted hair re-growth to a similar extent as minoxidil (Fig. 2, right panel). The skin color of HP-treated mice changed from pale to dark gray/black, indicating initiation of anagen.

HP also increases hair density and thickness in C57BL/6 mice Hair density and thickness were examined by dermoscopy. Figure 3a shows dermoscopic images of hair density and diameter at the $14^{\text {th }}$ and $16^{\text {th }}$ day after depilation. Hair density was higher in HP- or $5 \%$ minoxidiltreated mice than in normal saline-treated mice. A significant difference in hair density was observed at day 14; however, this difference decreased by at day 16 (Fig. 3a, b). Hair diameter was also larger in the HP- and $5 \%$ minoxidil-treated mice than in the mice treated with normal saline (Fig. 3a, c).

\section{HP enhanced the anagen induction and the cellular proliferation of hair follicle}

Histological examination showed that hair follicles in the normal saline-treated mice resided in the dermis, indicating that they had not fully developed to anagen (Fig. 4, upper left). However, the hair shafts of follicles in the skin of HP-treated mice extended through the epidermis, indicating that they were fully developed to anagen IV phase (Fig. 4, upper right). Immunohistochemical analysis confirmed that BrdU incorporation 


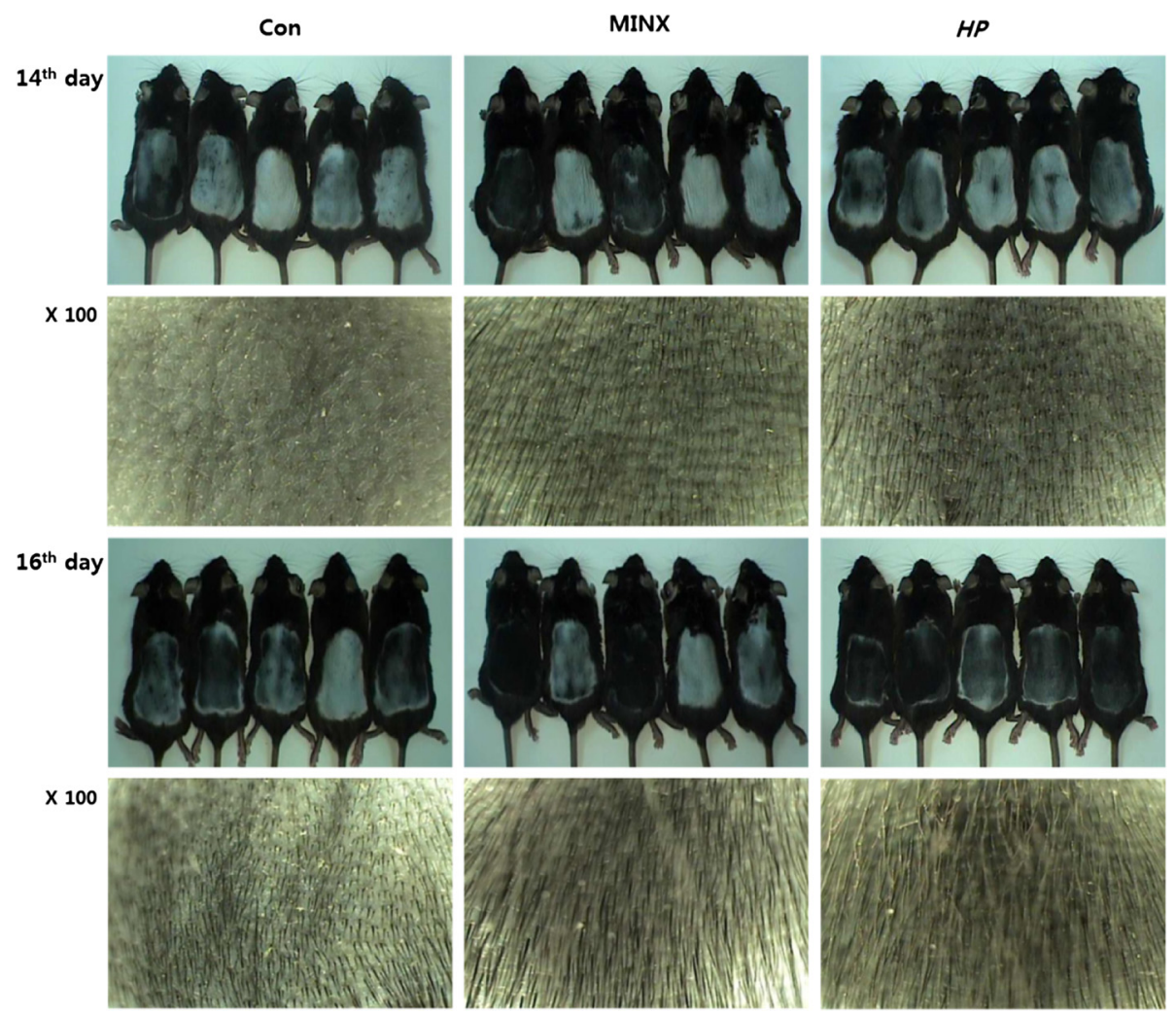

Fig. 2 Gross observation of hair re-growth in C57BL/6 mice. At the 14th and 16th days after depilation, skin color and hair growth of the depilated skin lesions were observed. Each group of mice has been topically treated with normal saline or $5 \%$ minoxidil or HP once a day for consecutive 15 days respectively. Depilated dorsal skin lesions were photographed at the 14th and 16th day after depilation. Hair regrowth, as well as skin darkness, was increased in mice treated with HP as compared to normal control mice

was prominently increased by HP (Fig. 4, middle right). Strongly positive reactions against BrdU were observed in the bulge, inner and outer root sheath, panniculus carnosus, and epidermal epithelium, indicating that HP induced active cell division in the hair follicle. HP also increased the expression of PCNA in the hair bulb, and in the inner and outer root sheath (Fig. 4, lower right, large box).

\section{HP upregulates the expression of fibroblast growth} factor-7 (FGF-7) involved in anagen development HP increased the expression of FGF-7 in the epidermis, and inner and outer root sheath, to a degree comparable to minoxidil treatment (Fig. 5a, right, large box). HP also increased the mRNA and protein expression of FGF-7, key molecule in tissue repair and anagen induction in the dorsal skin lesions of C57BL/6 mice (Fig. 5b).

\section{Discussion}

Placenta exerts critical roles in the development of fetus by exchanging nutrient, oxygen, waste products between mother and fetus, producing hormones which support the pregnancy. Placenta contains various bioactive substances including nucleic acid, amino acid [20]. Essential and non-essential amino acids contained in placenta were reported to be essential for the making fetal proteins [21]. Placenta has been used for the medical purpose since thousand years ago, in Eastern countries. In Traditional Chinese Medicine, human placenta (HP) has been used to enhance the vital essence [22]. Nowadays HP is considered as a source of cells with stem cell potential and could be able to applicate the tissue injuries [23]. Although a number of clinical studies about the beneficial effects of placenta extracts has been reported [24-27], the vast majority of the functional studies of the placental extracts was focused on it's wound healing potential in the tissue repairing process [28-31]. Placenta extract was reported to increase the expression of bFGF and TGF- $\beta 1$, two key factors involved in wound healing in the rat wound skin [14]. Aqueous extract of placenta was known to contain various peptides responsible for tissue regeneration [29]. Angiogenesis is a key process in the wound healing. New vessels deliver oxygen and nutrients to the injured lesions thereby facilitating tissue regeneration [32]. Angiogenic factor which suggested to be peptide was extracted from placenta 

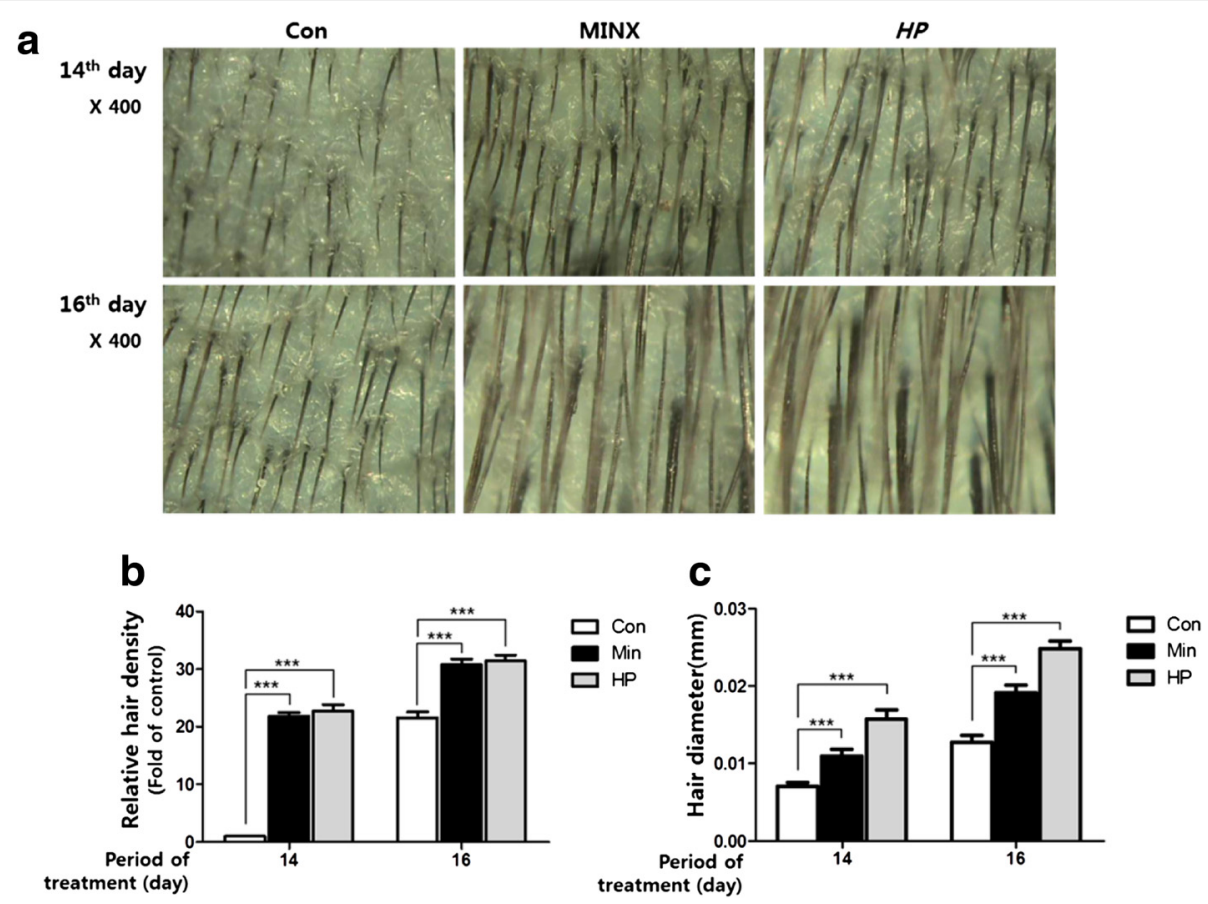

Fig. 3 Dermoscopic observation of hair regrowth in C57BL/6 mice. Dorsal skins of mice were photographed by dermoscopy at the 14th and 16th day after depilation. Hair density was expressed as the number of hairs per unit area. Compared to the skin of the mice in the normal saline-treated control group (Con), the skin of the mice in the $5 \%$ minoxidil (Min)- or HP-treated group had increased hair density and diameter (a). Hair density and hair diameter was greater in HP treated group than normal control group (b, c). Original Magnification: X100, X400. Values represent the mean \pm standard deviation. ${ }^{* *} P<0.001$, compared to control

[33]. Placenta extracts also induced nitric oxide (NO), a vasodilator in the mouse peritoneal macrophages [34]. More recently, placenta extract was also reported to inhibit Proteinase K, a microbial protease, thereby possibly prevent excessive proteolysis observed in bacterial infections [35].

Besides the wound healing, hair follicle cycle represents the other physiological regenerating event occurring in the skin. Growth factors involved in the wound healing are also involved in the hair follicle cycle [7]. Therefore, regenerative potential of placenta extract could also influence the hair cycle. Among the bioactive substance contained in placenta extract, several amino acids were known to positively influence hair growth. Arginine, a precursor of nitric oxide, plays an important role in skin angiogenesis [36]. Glutamine delivers sulphur which is necessary for hair growth [37]. Women with increased hair shedding responded to 1-lysine and iron therapy [38].

Hair follicles undergo successive growth, regression, rest, and shedding over the life time. Hair follicular cells including mesenchymal cells of dermal papilla and overlying epithelial cells which is responsible for making hair shaft only proliferate during anagen phase [6]. Therefore, length and thickness of each hair shafts largely determined by the duration of anagen. Hair follicle miniaturization, one of the most characteristic features of androgenic alopecia, was due to the progressive shortening of anagen in response to DHT [2]. Therefore, a rational strategy for the treatment of androgenic alopecia is to inhibit DHT activity or to prolong the duration of the anagen phase. Transition from anagen to categen is regulated by various molecules released from hair follicular cells. Among them Fibroblast growth factor-7 (FGF-7) was known to play a pivotal role in the reentering of hair follicle to the next anagen phase [7, 39]. FGF-7, also known as keratinocyte growth factor, which plays important role in the regulation of proliferation in epithelial tissues [40], also protects the hair follicle from cytotoxic agents or UV irradiation [41, 42].

In this study, we found that HP treatment accelerated anagen induction in the hair follicles of C57BL/6 mice. Gross observation showed that the capacity of HP to enhance hair growth in C57BL/6 mice was comparable to that of minoxidil. Histological examination revealed that hair follicles in normal saline-treated mice were smaller and less developed than those in minoxidil- or HP-treated mice. This observation is consistent with the gross examination indicating that HP has the potential to 


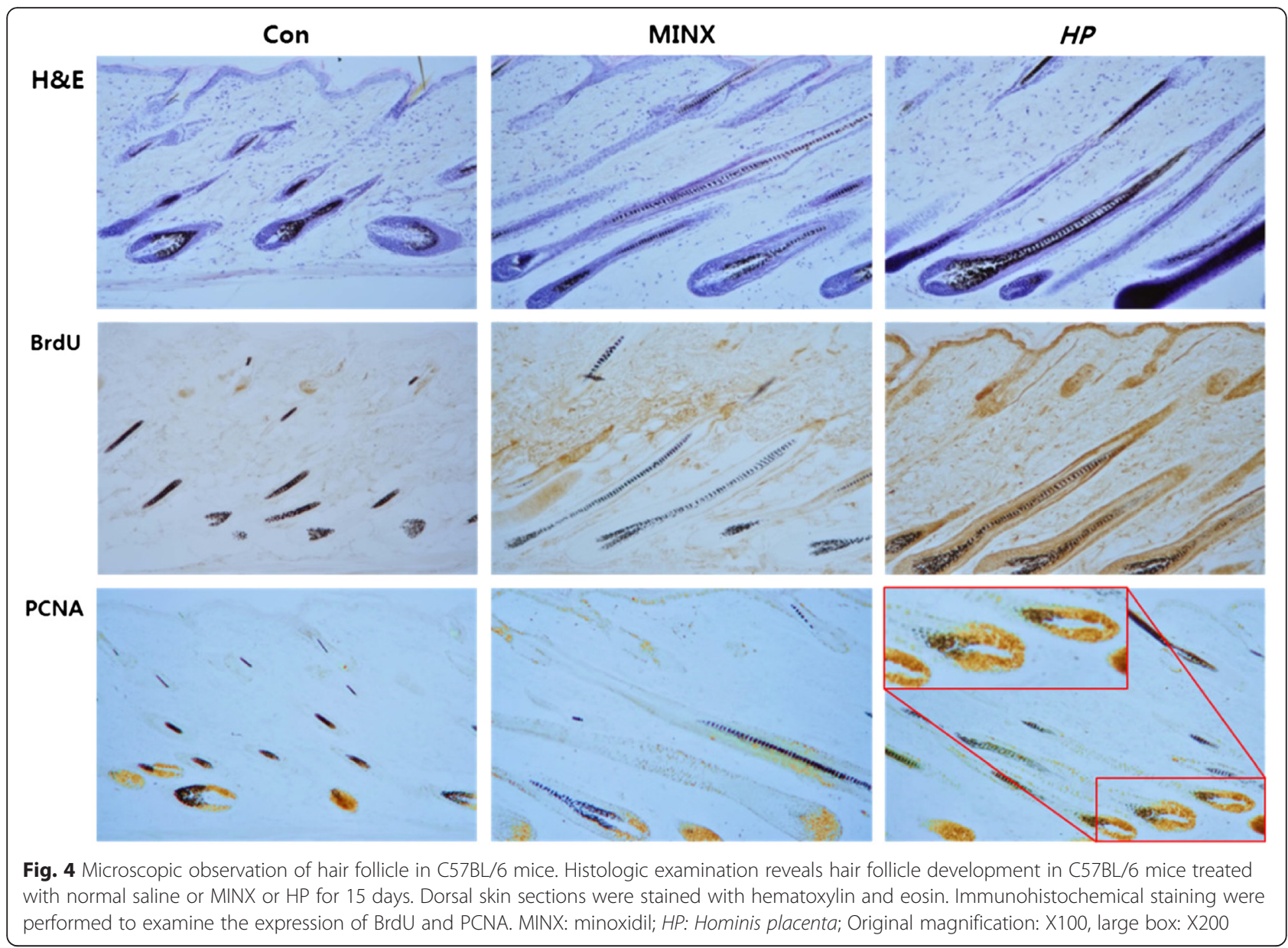

enhance anagen induction. As expected, hair density and diameter were also increased by the treatment of HP.

To evaluate the effect of HP on hair matrix cell proliferation during anagen, we examined the pattern of BrdU incorporation and PCNA expression. High levels of
BrdU incorporation were observed in the dermal papilla, outer layer of the hair shaft, and connective tissue. PCNA was highly expressed in the lower regions of the hair matrix, indicating that HP treatment induced DNA synthesis (Fig. 4, lower left). This finding is consistent

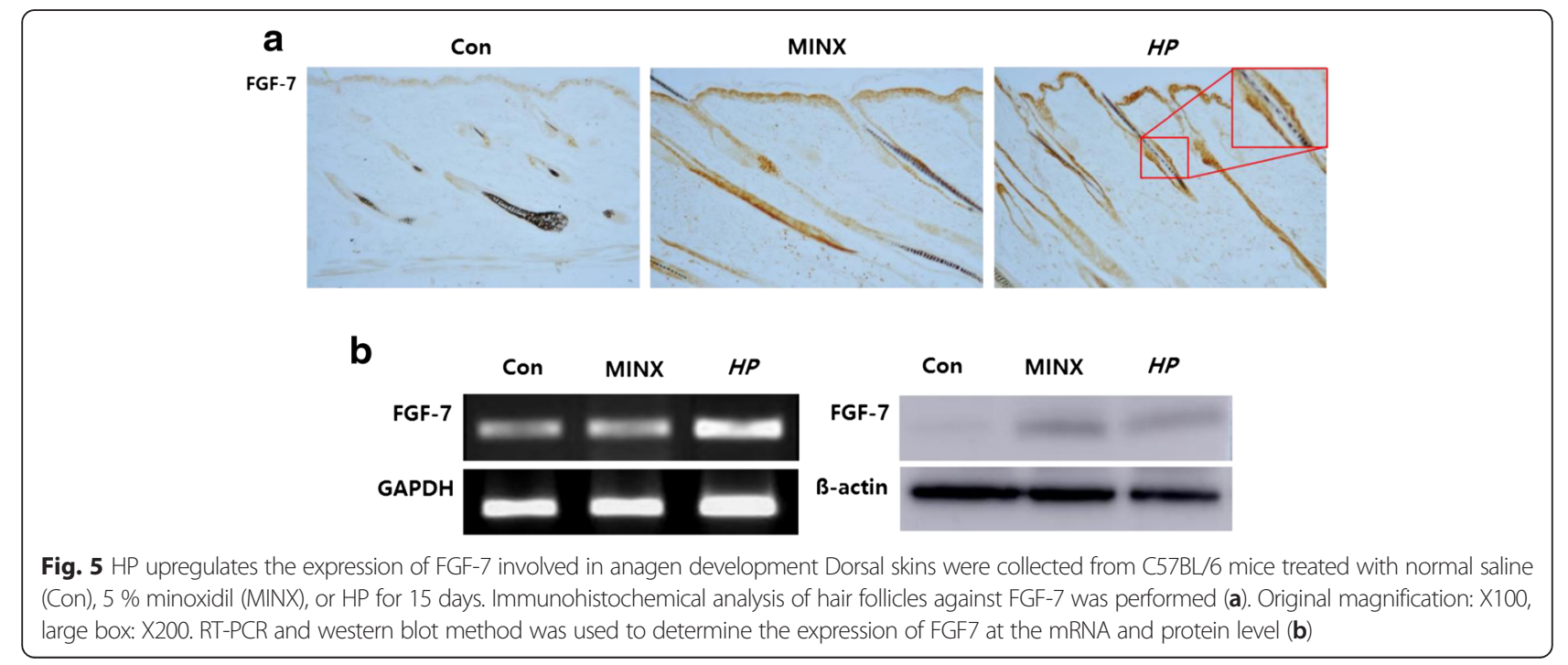


with the previous observation that HP increases hair diameter, because hair thickness is proportional to the volume of the hair matrix.

It is well known that the hair cycle is regulated by complex epithelial-mesenchymal interactions. A number of growth factors in the dermal papilla and overlying epithelial cells are known to interact with each other during hair growth cycle progression [43]. FGF-7 plays a pivotal role on the hair follicular cycle [44, 45]. We found that FGF-7 expression was increased in the outer layer of the hair shaft and surface epithelium by the treatment of HP. Increased expression of FGF-7 in the skin tissues both mRNA and protein level, determined by RT-PCR and western blot respectively.

We showed that topical treatment with HP induced robust hair re-growth in C57BL/6 mice owing to proliferation of hair matrix cells and upregulation of FGF-7. This study helps to understand the basic mechanism through which HP acts on hair follicle development. In particular, increased expression of FGF-7 by HP explains it's positive effects on the anagen induction.

\section{Conclusions}

HP has a potent hair growth-promoting activity; therefore, it may be a good candidate for the treatment of alopecia.

\section{Abbreviations}

$\mathrm{AA}$, androgenic alopecia; $\mathrm{BrdU}$, bromodeoxyuridine; $\mathrm{DHT}$, dihydrotestosterone: ECL, enhanced chemilumescent; FGF-7, fibroblast growth factor-7; HP, Hominis Placenta; PCNA, proliferating cell nuclear antigen; PVDF, polyvinylidene difluoride; RT-PCR, reverse transcriptase-polymerase chain reaction

\section{Acknowledgments}

This study was supported by the National Research Foundation of Korea (NRF) grant funded by the Korea government (MSIP) (2014R1A5A2009936).

\section{Funding}

This study was supported by the National Research Foundation of Korea (NRF) grant funded by the Korea government (MSIP) (2014R1A5A2009936).

\section{Availability of data and materials}

The datasets supporting the conclusions of this article are included within the article.

\section{Authors' contributions}

HSS and HSJ designed the study and HSJ wrote the manuscript. DJL and JHC conducted animal study. CHL and HRK contributed to microscopic data analysis. JEK and KTH helped the molecular analyses and improved the style of written English. MHJ helped to response the reviewer's comment and wrote parts of discussion. BJK provided the western data during revision. All authors read and approved the final manuscript.

\section{Competing interests}

The authors declare that they have no competing interests.

\section{Consent for publication}

This information is not relevant.

Ethics approval and consent to participate

This information is not relevant.

\section{Author details}

'Department of Ophthalmology, Otolaryngology and Dermatology, Korean Medicine Hospital, Pusan National University, Yangsan, Republic of Korea. 2Department of Clinical Medicine, Graduate School, Kyung Hee University, Seoul, Republic of Korea. ${ }^{3}$ Chung Jaeho Traditional Korean Medical Clinic, Seoul, Republic of Korea. ${ }^{4}$ Department of Anatomy, College of Korean Medicine, Woosuk University, Wanju, Republic of Korea. ${ }^{5}$ College of food \& biotechnology, Woosuk University, Wanju, Republic of Korea. ${ }^{6}$ Department of Pathology, College of Korean Medicine, Dongguk University, Goyang, Republic of Korea. ${ }^{7}$ Division of Longevity and Biofunctional Medicine, School of Korean Medicine, Pusan National University, Yangsan, Republic of Korea. ${ }^{8}$ Healthy Aging Korean Medical Research Center, School of Korean Medicine, Pusan National University, Yangsan, Republic of Korea. ${ }^{9}$ Division of Applied Medicine, School of Korean Medicine, Pusan National University, Yangsan, Republic of Korea.

Received: 7 July 2015 Accepted: 15 June 2016

Published online: 07 July 2016

\section{References}

1. Ellis JA, Stebbing M, Harrap SB. Polymorphism of the androgen receptor gene is associated with male pattern baldness. J Invest Dermatol. 2001; 116(3):452-5.

2. Kaufman KD. Androgens and alopecia. Mol Cell Endocrinol. 2002:198(1-2):89-95.

3. Hadshiew IM, Foitzik K, Arck PC, Paus R. Burden of hair loss: stress and the underestimated psychosocial impact of telogen effluvium and androgenetic alopecia. J Invest Dermatol. 2004;123(3):455-7.

4. Gilhar A, Keren A, Shemer A, d'Ovidio R, Ullmann Y, Paus R. Autoimmune Disease Induction in a Healthy Human Organ: A Humanized Mouse Model of Alopecia Areata. J Invest Dermatol. 2013;133(3):844-7.

5. Botchkarev VA, Komarova EA, Siebenhaar F, Botchkareva NV, Komarov PG, Maurer M, Gilchrest BA, Gudkov AV. p53 is essential for chemotherapyinduced hair loss. Cancer Res. 2000;60(18):5002-6.

6. Paus R, Cotsarelis G. The biology of hair follicles. New Engl I Med. 1999; 341(7):491-7.

7. Stenn KS, Paus R. Controls of hair follicle cycling. Physiol Rev. 2001;81(1):449-94.

8. Messenger AG, Rundegren J. Minoxidil: mechanisms of action on hair growth. Br J Dermatol. 2004;150(2):186-94.

9. Price VH. Treatment of hair loss. New Engl J Med. 1999;341(13):964-73.

10. Zhao L, Li JH, Zhu DZ, Ji BP. Principal component analysis of nutrients in five varieties of velvet antler (Cornu Cervi Pantotrichum). Guang pu. 2010; 30(9):2571-5.

11. Wang J, He G, Fu W, Su B, Wang W, Zhang Y, Lu Y, He M, Zhu Z. Extraction and sequencing of trace DNA from cornu Cervi pantotrichum. China J Chinese Meter Med. 1997;22(10):579-83. 638.

12. Zhang S, Li F. Determination of amino acids in cornu cervi pantotrichum of different specifications. China J Chinese Meter Med. 2013;38(12):1919-23.

13. Liu Y, Zhang GJ, Sun SQ, Noda I. Study on similar traditional Chinese medicines cornu Cervi pantotrichum, cornu Cervi and cornu Cervi degelatinatum by FT-IR and 2D-IR correlation spectroscopy. J Pharmaceut Biomed. 2010;52(4):631-5.

14. Wu CH, Chang GY, Chang WC, Hsu CT, Chen RS. Wound healing effects of porcine placental extracts on rats with thermal injury. Br J Dermatol. 2003; 148(2):236-45.

15. Seo TB, Han IS, Yoon JH, Seol IC, Kim YS, Jo HK, et al. Growth-promoting activity of Hominis Placenta extract on regenerating sciatic nerve. Acta Pharmacol Sin. 2006;27(1):50-8

16. Hauser GA. Placental Extract Injections in the Treatment of Loss of Hair in Women. Int J Tissue React. 1982:4(2):159-63.

17. Zhang D, Lijuan G, Jingjie L, Zheng L, Wang C, Wang Z, et al. Cow placenta extract promotes murine hair growth through enhancing the insulin - like growth factor-1. Indian J Dermatol. 2011;56(1):14-8.

18. Nowakowski RS, Lewin SB, Miller MW. Bromodeoxyuridine immunohistochemical determination of the lengths of the cell cycle and the DNA-synthetic phase for an anatomically defined population. J Neurocytol. 1989;18(3):311-8.

19. Ito M, Kizawa K, Hamada K, Cotsarelis G. Hair follicle stem cells in the lower bulge form the secondary germ, a biochemically distinct but functionally equivalent progenitor cell population, at the termination of catagen. Differentiation. 2004;72(9-10):548-57.

20. Shibasaki T, Odagiri E, Shizume K, Ling N. Corticotropin-Releasing Factor-Like Activity in Human Placental Extracts. J Clin Endocr Metab. 1982;55(2):384-6. 
21. Cleal JK, Lewis RM. The mechanisms and regulation of placental amino acid transport to the human foetus. J Neuroendocrinol. 2008;20(4):419-26.

22. Yang GQ, Zou XH. Research Advances on Chemical Compositions, Pharmacological Effect and Clinic Application of Placenta and Its Extract from Human and Animals. J Shenyang Agri U. 2003;34:150-154.

23. Lange-Consiglio A, Corradetti B, Bizzaro D, Magatti M, Ressel L, Tassan S, et al. Characterization and potential applications of progenitor-like cells isolated from horse amniotic membrane. J Tissue Eng Regen M. 2012;6(8):622-35.

24. Kaushal V, Verma K, Manocha S, Hooda HS, Das BP. Clinical evaluation of human placental extract (placentrex) in radiation-induced oral mucositis. Int J Tissue React. 2001;23(3):105-10

25. Chandanwale A, Langade D, Mohod V, Sinha S, Ramteke A, Bakhshi GD, et al. Comparative evaluation of human placental extract for its healing potential in surgical wounds after orthopaedic surgery: an open, randomised, comparative study. J Indian Med Assoc. 2008;106(6):405-8.

26. Hijikata Y, Kano T, Xi L. Treatment for intractable anemia with the traditional Chinese medicines Hominis Placenta and Cervi Cornus Colla (deer antler glue). Inter J Gen Med. 2009;2:83-90.

27. Choi JY, Lee K, Lee SM, Yoo SH, Hwang SG, Lee SW, et al. Efficacy and safety of human placental extract for alcoholic and nonalcoholic steatohepatitis: an openlabel, randomized, comparative study. Biol Pharm Bull. 2014;37(12):1853-9.

28. Datta P, Bhattacharyya D. Spectroscopic and chromatographic evidences of NADPH in human placental extract used as wound healer. J Pharmaceut Biomed. 2004;34(5):1091-8.

29. Chakraborty PD, Bhattacharyya D. Isolation of fibronectin type III like peptide from human placental extract used as wound healer. J Chromatogr B. 2005;818(1):67-73.

30. Chakraborty PD, De D, Bandyopadhyay S, Bhattacharyya D. Human aqueous placental extract as a wound healer. J Wound Care. 2009;18(11):462. 464-467.

31. De D, Chakraborty PD, Bhattacharyya D. Analysis of free and bound NADPH in aqueous extract of human placenta used as wound healer. J Chromatogr B. 2009:877(24):2435-42.

32. Logan A, Weatherhead B. Effects of alpha-melanocyte-stimulating hormone and [8-arginine]-vasotocin upon melanogenesis in hair follicle melanocytes in vitro. J Endocrinol. 1981;91(3):501-7.

33. Burgos $\mathrm{H}$. Angiogenic factor from human term placenta. Purification and partial characterization. Eur J Clin Invest. 1986;16(6):486-93.

34. Chakraborty PD, Bhattacharyya D, Pal S, Ali N. In vitro induction of nitric oxide by mouse peritoneal macrophages treated with human placental extract. Int Immunopharmacol. 2006;6(1):100-7.

35. Sharma K, Mukherjee C, Roy S, De D, Bhattacharyya D. Human placental extract mediated inhibition of proteinase $\mathrm{K}$ : implications of heparin and glycoproteins in wound physiology. J Cell Physiol. 2014;229(9):1212-23.

36. Joshi M, Fuller LR, Batchelor GC. L-arginine metabolites regulate DNA synthesis and nitric oxide synthase activity in cultured human dermal microvascular endothelial cells-potential positive and negative regulators of angiogenesis derived from L-arginine. Cancer Invest. 1999;17(4):235-44.

37. Stuehr DJ, Gross SS, Sakuma I, Levi R, Nathan CF. Activated murine macrophages secrete a metabolite of arginine with the bioactivity of endothelium-derived relaxing factor and the chemical reactivity of nitric oxide. J Exp Med. 1989;169(3):1011-20.

38. Rushton DH, Ramsay ID, James KC, Norris MJ, Gilkes JJH. Biochemical and Trichological Characterization of Diffuse Alopecia in Women. Br J Dermatol. 1990;123(2):187-97.

39. Werner S, Grose R. Regulation of wound healing by growth factors and cytokines. Physiol Rev. 2003;83(3):835-70.

40. Housley RM, Morris CF, Boyle W, Ring B, Biltz R, Tarpley JE, et al. Keratinocyte growth factor induces proliferation of hepatocytes and epithelial cells throughout the rat gastrointestinal tract. J Clin Invest. 1994;94(5):1764-77.

41. Booth C, Potten CS. Keratinocyte growth factor increases hair follicle survival following cytotoxic insult. J Invest Dermatol. 2000;114(4):667-73.

42. Braun S, Krampert M, Bodo E, Kumin A, Born-Berclaz C, Paus R, et al Keratinocyte growth factor protects epidermis and hair follicles from cell death induced by UV irradiation, chemotherapeutic or cytotoxic agents. J Cell Sci. 2006;119(23):4841-9.

43. Botchkarev VA, Kishimoto J. Molecular control of epithelial-mesenchymal interactions during hair follicle cycling. J Invest Derm Symp P. 2003:8(1):46-55.

44. Rosenquist TA, Martin GR. Fibroblast growth factor signalling in the hair growth cycle: expression of the fibroblast growth factor receptor and ligand genes in the murine hair follicle. Dev Dynam. 1996;205(4):379-86.
45. Kawano M, Komi-Kuramochi A, Asada M, Suzuki M, Oki J, Jiang J, et al Comprehensive analysis of FGF and FGFR expression in skin: FGF18 is highly expressed in hair follicles and capable of inducing anagen from telogen stage hair follicles. J Invest Dermatol. 2005;124(5):877-85.

\section{Submit your next manuscript to BioMed Central and we will help you at every step:}

- We accept pre-submission inquiries

- Our selector tool helps you to find the most relevant journal

- We provide round the clock customer support

- Convenient online submission

- Thorough peer review

- Inclusion in PubMed and all major indexing services

- Maximum visibility for your research

Submit your manuscript at www.biomedcentral.com/submit
C Biomed Central 